\title{
Nonlinear Models for Relativity Effects in Electromagnetism
}

\author{
Santosh Devasia \\ Mechanical Engineering, University of Washington, Seattle, WA 98195-2600, USA \\ Reprint requests to S. D.; Fax: +1-206-685-8047; E-mail: devasia@u.washington.edu \\ Z. Naturforsch. 64a, 327 - 340 (2009); received July 2, 2008 / revised October 13, 2008 \\ Experimental energy measurements tend to be smaller than the predicted values in (i) the absorp- \\ tion of high-energy particles (in cloud chambers) and (ii) the average energy determination of the \\ classical $\beta$-ray spectrum of radium E (using magnetic fields). To address these differences in energy \\ measurements, we reconsider relative-velocity-dependent models in electromagnetism proposed ini- \\ tially by Weber before data from cathode-ray-tube (CRT) experiments was available. It is shown that \\ identifying the nonlinear, relative-velocity terms using CRT data results in a model, which (i) cap- \\ tures relativity effects in optics and high-energy particles, and (ii) explains the apparent discrepancies \\ in experimental energy measurements.
}

Key words: Relative Velocity; Weber; Relativity; Electromagnetism.

\section{Introduction}

Relative-velocity-dependent models in electromagnetism were proposed initially by Weber $[1,2]$ before data from cathode-ray-tube (CRT) experiments was available. In contrast, a nonlinear relative-velocitydependent model is developed in the present article, that is based on data from CRT experiments. This article addresses two challenges in the use of such relativevelocity-dependent models: (i) to capture both lowvelocity and high-velocity effects in electromagnetism; and (ii) to maintain model invariance between inertial reference frames. The first challenge is addressed by using the nonlinearity of the proposed model to capture: (a) low-velocity effects such as the force between two current-carrying wires; and (b) high-velocity effects such as the mass increase seen in CRT experiments. The second challenge, to maintain model invariance between different inertial frames, is addressed by accounting for the relative-velocity effects in Lorentz and Maxwell's equations. The resulting model not only captures relativity effects in optics and highenergy particles but also explains apparent discrepancies between predicted and measured energy in: (i) the absorption of high-energy particles in cloud chambers [3]; and (ii) the average energy determination of the $\beta$-ray spectrum using magnetic fields [4-7].

Measurements of the energy lost by high-energy electrons due to absorption in lead, by Crane and co-workers, tend to be more than $50 \%$ of the ex- pected value [3]. Studies of possible electron scattering and potential increase in path length (through the lead) could not explain this discrepancy [8]. The inability to resolve this discrepancy led Richardson and Kurie [9] to conclude that the cloud-chamberabsorption method is not reliable for energy measurements "even when applied by careful investigators". However, the discrepancy in the measured energy can be accounted for by the model presented in the present article. The proposed model also explains apparent discrepancies between predicted and measured energies in classical $\beta$-ray-spectrum experiments [4-7]. Ellis and Wooster [4] found the average disintegration energy of radium $\mathrm{E}$ ( $\mathrm{Ra} \mathrm{E}$ ) to be $0.344 \mathrm{MeV}$ using temperature measurements while reporting that the average energy found by Madgwick from the $\beta$-ray spectrum was $0.395 \mathrm{MeV}$. Moreover, Madgwick's data (including the location of the spectrum's peak) was confirmed independently by Ho and Wang [7]. The difference in the average energy between these two different approaches led to the development of several correction factors for potential errors in measurements at the lower end of the $\beta$-ray spectrum for reconciling the difference $[10,11]$. In contrast, the predicted average energy of the $\beta$-ray spectrum using the proposed relativevelocity approach matches the value from temperature measurements by Ellis and Wooster [4], without the need for correction factors.

The proposed relative-velocity model has the following general form for the Lorentz force $F_{E}$ on a par- 
ticle of charge $q$ due to an electric field $E$ :

$$
F_{E}=\left[\mathcal{N}_{\perp}\left(v_{\text {rel }}\right)\right] q E_{\perp}+\left[\mathcal{N}_{\|}\left(v_{\text {rel }}\right)\right] q E_{\|},
$$

where $E_{\perp}$ and $E_{\|}$are the components of the field perpendicular and parallel to the velocity $v_{\text {rel }}$ between the field and the particle. It has to be noted that adhoc choices of the nonlinearities $\left[\mathcal{N}_{\perp}, \mathcal{N}_{\|}\right.$in (1)] are not acceptable. For example, instead of a velocitydependent increase in mass, $m=m_{0} / \sqrt{1-\frac{\left|v_{\text {rel }}\right|^{2}}{c^{2}}}$, a reduction of the Lorentz force, such as $\mathcal{N}_{\perp}=$ $\sqrt{1-\frac{\left|\nu_{\text {rel }}\right|^{2}}{c^{2}}}$, might be considered to match the relativistic, velocity-dependent increase in mass in CRT experiments (where $c$ is the speed of light and $m_{0}$ is the rest mass). However, it is shown that such a nonlinearity is not consistent with low-speed effects such as Ampere's law for the force between two current-carrying wires.

In the present article, the form of the perpendicular nonlinearity $\mathcal{N}_{\perp}$ [in (1)] is identified using: (i) the relativistic mass increase in CRT experiments; and (ii) conservation of the field's energy density at low speeds. It is shown that the resulting form of the perpendicular nonlinearity $\mathcal{N}_{\perp}$ also uniquely identifies: (i) the kinetic energy of a particle; and (ii) the parallel nonlinearity $\mathcal{N}_{\|}$. In addition to matching the relativistic mass increase in CRT experiments (as expected, since the nonlinearity $\mathcal{N}_{\perp}$ is identified using CRT observations), the resulting nonlinearity expression also matches the low-speed Ampere's law for the force between two current-carrying wires. Furthermore, the resulting energy expression explains the apparent discrepancies in the measured energy in high-energy electron absorption [3] and $\mathrm{Ra}$ E disintegration [5].

The second difficulty, to find appropriate transformations to relate observations in different inertial frames, can be addressed by the proposed relativevelocity-based approach where spatial velocity distributions $\left(V_{E}, V_{B}\right)$ are assigned to the electrical field $E$ and magnetic field $B$. It is shown that Maxwell's equations, if adapted to include these relative-velocity distributions, are still co-ordinate invariant. The effects of the proposed approach on the propagation of light and the explanation of optical phenomena are considered in this article. Most importantly, the relative-velocity approach models relativistic effects such as the transverse Doppler effect and the convection of light by moving media (Fresnel drag). Thus, the article presents a Weber-type relative-velocity-dependent modeling approach that: (i) captures relativistic effects in optics and high-energy particles; and (ii) explains apparent discrepancies in experimental energy measurements.

\section{Relative-Velocity Approach}

In this section, the nonlinearities $\mathcal{N}_{\perp}, \mathcal{N}_{\|}$[in (1)] are identified. Low-speed effects (energy density invariance) and high-speed (CRT) effects are considered in the first two subsections to identify the perpendicular nonlinearity $\mathcal{N}_{\perp}$, which is then used in the third subsection to identify expressions for the kinetic energy and the parallel nonlinearity $\mathcal{N}_{\|}$.

\subsection{Energy Density Conservation at Low Speeds}

In an inertial frame $O$, let velocity fields $V_{E}$ and $V_{B}$ be associated with the electric field $E$ and magnetic field $B$, respectively. These are the velocities of the electric and magnetic fields relative to the inertial frame $O$. Then, the Lorentz force between a charged particle $q$, moving with velocity $V$ relative to the inertial frame $O$, and the fields is modeled as a function of the relative velocity between the particle and the field as proposed by Weber [1,2], and generalized in the present article. The main idea is that a moving electric field introduces an apparent magnetic field (and vice versa); the model maintains a constant total energy density (electric and magnetic) that is independent of the relative velocity. This subsection begins with the relative-velocity-dependent modeling of the magnetic field.

\section{Low-Speed, Relative-Velocity Modeling of the Magnetic Field}

The Lorentz force on an electrical charge $q$ due to the magnetic field in terms of the relative velocity $(V-$ $V_{B}$ ) of the particle with respect to the field is

$$
F_{B}=q\left(V-V_{B}\right) \times B .
$$

Thus, the magnetic field $B$ appears to have an effective electric field $E_{B}$, perpendicular to the relative velocity $\left(V-V_{B}\right)$, given by

$$
E_{B}=\left(V-V_{B}\right) \times B .
$$

This apparent electric field implies that the field energy would vary with the relative velocity of the charged particle in the same reference frame. To avoid such variation, a reduction of the apparent magnetic field $B_{B}$ 
in the perpendicular direction is considered so that the net energy of the apparent fields is independent of the relative velocity. In particular, it is assumed that the effective magnetic field (acting on an ideal magnetic particle that is moving with velocity $V$ according to observer $O$ ) is given by

$$
B_{B}=B_{\|}+\gamma_{B} B_{\perp},
$$

where $\gamma_{B} B_{\perp}$ is the vector component of the magnetic field perpendicular to the relative velocity $\left(V-V_{B}\right)$, and $B_{\|}$is the vector component of the magnetic field parallel to the relative velocity $\left(V-V_{B}\right)$. In the nominal case, when the relative velocity is zero, i. e., $V=V_{B}$, we have no change in the perpendicular component and therefore $\gamma_{B}=1$ for this case. When the relative velocity is nonzero, the factor $\gamma_{B}$ is chosen such that the net energy density of $B_{B}$ and $E_{B}$ (due to the magnetic field $B)$ is independent of the relative velocity $\left(V-V_{B}\right)$. Moreover, the only variations in the fields are in the perpendicular components. Therefore, by matching the energy density in the field's perpendicular component for the case that the relative velocity is nonzero to the case if the relative velocity is zero, one obtains

$$
\frac{\gamma_{B}^{2}}{2 \mu}\left|B_{\perp}\right|^{2}+\frac{\varepsilon}{2}\left|E_{B}\right|^{2}=\frac{1}{2 \mu}\left|B_{\perp}\right|^{2},
$$

where $|\cdot|$ represents the magnitude of a vector, $\varepsilon$ is the permittivity, and $\mu$ is the permeability. Substituting the apparent electric field $E_{B}$ from (3), i. e.,

$$
E_{B}=\left(V-V_{B}\right) \times B=\left(V-V_{B}\right) \times B_{\perp},
$$

into (5), yields

$$
\frac{\gamma_{B}^{2}}{2 \mu}\left|B_{\perp}\right|^{2}+\frac{\varepsilon\left|V-V_{B}\right|^{2}}{2}\left|B_{\perp}\right|^{2}=\frac{1}{2 \mu}\left|B_{\perp}\right|^{2}
$$

and

$$
\gamma_{B}=\sqrt{1-\frac{\left|V-V_{B}\right|^{2}}{c^{2}}}=\sqrt{1-\beta_{B}^{2}},
$$

where $c=\sqrt{1 / \varepsilon \mu}$ is the speed of light and $\beta_{B}$ is the normalized relative speed:

$$
\beta_{B}=\left|V-V_{B}\right| / c .
$$

Thus, an electric particle moving with the velocity $V$ is affected by the electric field $E_{B}$; a magnetic particle moving with the velocity $V$ is affected by the magnetic field $B_{B}$ and the electric field $E_{B}$ moving with velocity $V_{B}$.

Low-Speed, Relative-Velocity Modeling of the Electric Field

Similar to the last subsection, an electric field $E$ appears to have an effective magnetic field $B_{E}$, perpendicular to the relative velocity $\left(V-V_{E}\right)$, given by

$$
B_{E}=-\varepsilon \mu\left(V-V_{E}\right) \times E,
$$

where the term $-\varepsilon \mu$ is used to match the magnetic field produced by a current-carrying wire (Ampere's law). In particular, if $\rho$ (charge per unit length) is flowing with velocity $v$ through a wire (which is stationary in the reference frame $O$ ), then the electric field $E_{\rho}$ associated with this charge, at a distance $r \hat{r}$ from the wire, is given by $E_{\rho}=[\rho /(2 \pi \varepsilon r)] \hat{r}$, where $\hat{r}$ represents a unit direction vector. Note that the velocity associated with this electric field is the velocity $v$ of the charge flowing through the wire. Therefore, from (10), the magnetic field $B_{\rho}$ at a distance $r \hat{r}$ from the wire is

$$
\begin{aligned}
B_{\rho} & =-(\varepsilon \mu)(0-v) \times E_{\rho}=(\varepsilon \mu) v \times E_{\rho} \\
& =\varepsilon \mu[\rho /(2 \pi \varepsilon r)]|v| \hat{v} \times \hat{r} \\
& =[\mu I /(2 \pi r)] \hat{v} \times \hat{r},
\end{aligned}
$$

where $I$ is the current in the wire; this is the expression for a magnetic field produced by a current-carrying wire.

To keep the net energy independent of the relative velocity $\left(V-V_{E}\right)$, the following reduction $\gamma_{E}$ in the perpendicular direction of the apparent electric field $E_{E}$ is considered:

$$
E_{E}=E_{\|}+\gamma_{E} E_{\perp},
$$

where $\gamma_{E} E_{\perp}$ is the vector component of the electric field perpendicular to the relative velocity $\left(V-V_{E}\right)$, $E_{\|}$is the vector component of the electric field parallel to the relative velocity $\left(V-V_{E}\right)$, and the scaling factor $\gamma_{E}=1$, if the relative velocity is zero, i. e., $V=V_{E}$. The scaling factor $\gamma_{E}$ is obtained by equating the total energy density to the energy density of the electric field alone, if the relative velocity is zero, as

$$
\frac{\varepsilon \gamma_{E}^{2}}{2}\left|E_{\perp}\right|^{2}+\frac{1}{2 \mu}\left|B_{E}\right|^{2}=\frac{\varepsilon}{2}\left|E_{\perp}\right|^{2} .
$$

Substituting the apparent magnetic field $B_{E}$ from (10), i.e.,

$$
B_{E}=-\varepsilon \mu\left(V-V_{E}\right) \times E=-\varepsilon \mu\left(V-V_{E}\right) \times E_{\perp},
$$


into (13), yields

$$
\frac{\varepsilon \gamma_{E}^{2}}{2}\left|E_{\perp}\right|^{2}+\frac{\varepsilon^{2} \mu^{2}\left|V-V_{E}\right|^{2}}{2 \mu}\left|E_{\perp}\right|^{2}=\frac{\varepsilon}{2}\left|E_{\perp}\right|^{2}
$$

and a scaling factor

$$
\gamma_{E}=\sqrt{1-\frac{\left|V-V_{E}\right|^{2}}{c^{2}}} .
$$

This expression is similar to the one for the scaling factor for a magnetic field in (8). Thus, a magnetic particle moving with the velocity $V$ is affected by the magnetic field $B_{E}$; an electric particle moving with the velocity $V$ is affected by the electric field $E_{E}$ and the magnetic field $B_{E}$ moving with the velocity $V_{E}$. In particular, the net force on an electric particle (of charge $q$ ) is given by [from (2), (10) and (12)]

$$
\begin{aligned}
F_{E} & =q\left(V-V_{E}\right) \times B_{E}+q E_{\|}+q \gamma_{E} E_{\perp} \\
& =q\left(V-V_{E}\right) \times\left\{-\varepsilon \mu\left(V-V_{E}\right) \times E\right\}+q E_{\|}+q \gamma_{E} E_{\perp} \\
& =q \frac{\left|V-V_{E}\right|^{2}}{c^{2}} E_{\perp}+q E_{\|}+q E_{\perp} \sqrt{1-\frac{\left|V-V_{E}\right|^{2}}{c^{2}}} \\
& =q\left[\beta_{E}^{2}+\sqrt{1-\beta_{E}^{2}}\right] E_{\perp}+q E_{\|} \\
& =q \alpha E_{\perp}+q E_{\|},
\end{aligned}
$$

where the normalized relative speed $\beta_{E}$ and the scaling factor $\alpha$ are given by

$$
\beta_{E}=\left|V-V_{E}\right| / c \text { and } \alpha=\beta_{E}^{2}+\sqrt{1-\beta_{E}^{2}} .
$$

If the relative velocity is small, i.e., $\beta_{E}$ is small, the scaling factor $\alpha$ in the perpendicular force component of (17)

$$
F_{E, \perp}=q \alpha E_{\perp}
$$

can be simplified to

$$
\alpha=\beta_{E}^{2}+\sqrt{1-\beta_{E}^{2}} \approx 1+\frac{1}{2} \beta_{E}^{2} .
$$

Therefore, the simplified force on an electric particle in (17) becomes

$$
F_{E} \approx q\left(1+\frac{1}{2} \beta_{E}^{2}\right) E_{\perp}+q E_{\|} .
$$

\section{Saturation Effect}

The discussion in the present article is limited to the case that the magnitude of the relative velocity is less than the speed of light $c$, i. e., $\beta_{E} \leq 1$ and $\beta_{B} \leq 1$. The approach can be extended to higher relative speeds by fixing (saturating) the scaling factors to the values for the case if the relative speed equals the speed of light. For example, $\alpha$ in (18) is held constant for higher relative speeds $\beta_{E}>1$ as

$$
\alpha=1 \forall \beta_{E}>1 .
$$

Although not stated explicitly, equations are presented only for the case if $\beta_{E} \leq 1$ and $\beta_{B} \leq 1$ in the rest of the article.

\subsection{High-Speed Effects in the Relative-Velocity Model}

The relativistic mass dependence on speed is modeled as a slip effect, where the force on the particle reduces as the relative velocity increases. In particular, consider the augmentation of the Lorentz force on an electric particle, in (2) and (17), with relative-velocity terms $s_{\perp}$ and $s_{\|}$as

$$
\begin{gathered}
F_{B}=\left[s_{\perp}\left(\beta_{B}\right)\right] q\left(V-V_{B}\right) \times B_{\perp}, \\
F_{E}=\left[s_{\perp}\left(\beta_{E}\right)\right] q \alpha E_{\perp}+\left[s_{\|}\left(\beta_{E}\right)\right] q E_{\|}=F_{E, \perp}+F_{E, \|} \cdot
\end{gathered}
$$

The perpendicular and parallel slip terms $s_{\perp}$ and $s_{\|}$are identified in this subsection.

\section{Matching Cathode-Ray-Tube Observations}

Consider the forces on a charge, moving with the velocity $V$ perpendicular to a stationary magnetic field $B$ and electric field $E$, as in cathode-ray-tube (CRT) experiments [12]. These forces can be written, from (22) and (23), as

$$
\begin{aligned}
& F_{B}=s_{\perp}(\beta) q V \times B, \\
& F_{E}=s_{\perp}(\beta) \alpha(\beta) q E,
\end{aligned}
$$

where $\beta=\frac{|V|}{c}$. If the fields act on the charged CRT particle over some length $L$, then the change in velocity of the CRT particle along the application of the force during the time interval $\Delta t=L /|V|$ is given by

$$
\frac{F_{B} L}{m|V|} \text { and } \frac{F_{E} L}{m|V|},
$$

where $m$ is the mass of the particle (electron). Therefore, the change in angles $\left(\theta_{B}\right.$ and $\left.\theta_{E}\right)$ of the CRT particle's path along the action of the fields $B$ and $E$, respectively, can be approximated by using (24) and (25) 
as [12]

$$
\begin{gathered}
\theta_{B}=\left|\frac{F_{B} L}{m|V|^{2}}\right|=\frac{s_{\perp}(\beta) q|V||B| L}{m|V|^{2}}=\frac{s_{\perp}(\beta)|B| L}{\frac{m}{q}|V|}, \\
\theta_{E}=\left|\frac{F_{E} L}{m|V|^{2}}\right|=\frac{s_{\perp}(\beta) \alpha(\beta)|E| L}{\frac{m}{q}|V|^{2}} .
\end{gathered}
$$

In the absence of the relative velocity terms [i.e., $s_{\perp}(\beta)=1$ and $\alpha(\beta)=1$ ], a velocity-dependent mass variation can be used to explain the CRT data. In particular, the estimated velocity $V_{\mathrm{CRT}}$ and the estimated mass-to-charge ratio

$$
\frac{m\left(\beta_{\mathrm{CRT}}\right)}{q}=\frac{m}{q} \Psi\left(\beta_{\mathrm{CRT}}\right),
$$

with

$$
\beta_{\mathrm{CRT}}=\frac{\left|V_{\mathrm{CRT}}\right|}{c},
$$

from the CRT experiments would be related by

$$
\begin{aligned}
\theta_{B} & =\frac{|B| L}{\frac{m}{q} \Psi\left(\beta_{\mathrm{CRT}}\right)\left|V_{\mathrm{CRT}}\right|}, \\
\theta_{E} & =\frac{|E| L}{\frac{m}{q} \Psi\left(\beta_{\mathrm{CRT}}\right)\left|V_{\mathrm{CRT}}\right|^{2}},
\end{aligned}
$$

where $\Psi\left(\beta_{\mathrm{CRT}}\right)$ represents the CRT-predicted variation of mass with velocity. Dividing (26) and (27) by (29) and (30), respectively, yields

$$
\begin{aligned}
& s_{\perp}(\beta)=\frac{|V|}{\left|V_{\mathrm{CRT}}\right|} \frac{1}{\Psi\left(\beta_{\mathrm{CRT}}\right)}, \\
& s_{\perp}(\beta) \alpha(\beta)=\frac{|V|^{2}}{\left|V_{\mathrm{CRT}}\right|^{2}} \frac{1}{\Psi\left(\beta_{\mathrm{CRT}}\right)} .
\end{aligned}
$$

The velocity $V_{\mathrm{CRT}}$ predicted by the CRT experiments can be obtained by dividing (31) by (32) to obtain

$$
\left|V_{\mathrm{CRT}}\right|=\frac{|V|}{\alpha(\beta)} \text { or } \beta_{\mathrm{CRT}}=\frac{\beta}{\alpha(\beta)} .
$$

Furthermore, the perpendicular slip term $s_{\perp}(\beta)$ can be found by dividing the square of (31) by (32) and then substituting $\beta_{\mathrm{CRT}}$ from (33) to obtain

$$
s_{\perp}(\beta)=\frac{\alpha(\beta)}{\Psi\left(\beta_{\mathrm{CRT}}\right)}=\frac{\alpha(\beta)}{\Psi\left(\frac{\beta}{\alpha(\beta)}\right)} .
$$

Case 1: Matching the Relativistic Mass-Velocity Relation

The perpendicular term $s_{\perp}(\beta)$ can be chosen, as in (34), to exactly match the observed velocitydependent variation $\Psi$ in mass. In particular, if the CRT-predicted mass increase is given by the relativistic expression

$$
\Psi\left(\beta_{\mathrm{CRT}}\right)=\frac{1}{\sqrt{1-\beta_{\mathrm{CRT}}^{2}}},
$$

then the expression for the slip term $s_{\perp}$ is obtained, from (33) and (34), as

$$
\begin{aligned}
s_{\perp}(\beta) & =\alpha(\beta)\left\{\sqrt{1-\left[\frac{\beta}{\alpha(\beta)}\right]^{2}}\right\} \\
& =\sqrt{[\alpha(\beta)]^{2}-\beta^{2}} .
\end{aligned}
$$

\section{Case 2: Simplified Perpendicular Slip Term}

Consider the following, simplified expression $\bar{s}_{\perp}$ for the slip term $s_{\perp}(\beta)$ :

$$
\bar{s}_{\perp}(\beta)=\left[1-\beta^{8}\right]^{1 / 4} .
$$

This term does not lead to an exact match of the relativistic mass increase; however, it closely approximates the expression for the relativistic mass increase. In particular, assuming this form $\bar{s}_{\perp}(\beta)$ for the slip term, the velocity $\bar{\beta}_{\mathrm{CRT}}$ estimated in the CRT experiment, as in (33), is given by

$$
\bar{\beta}_{\mathrm{CRT}}=\frac{\beta}{\alpha(\beta)}=\frac{\beta}{\beta^{2}+\sqrt{1-\beta^{2}}} .
$$

Moreover, the apparent mass variation $\bar{\Psi}$ in the CRT experiment, as in (34), is given by

$$
\bar{\Psi}\left(\bar{\beta}_{\mathrm{CRT}}\right)=\frac{\alpha(\beta)}{s(\beta)}=\frac{\beta^{2}+\sqrt{1-\beta^{2}}}{\left[1-\beta^{8}\right]^{1 / 4}} .
$$

It has to be noted that the variation of $\bar{\Psi}\left(\bar{\beta}_{\mathrm{CRT}}\right)$ with velocity $\bar{\beta}_{\mathrm{CRT}}$ in (38) and (39), which would be obtained from a CRT experiment, is similar to the relativistic variation

$$
\Psi\left(\bar{\beta}_{\mathrm{CRT}}\right)=\frac{1}{\sqrt{1-\bar{\beta}_{\mathrm{CRT}}^{2}}},
$$

as shown in Figure 1. 
(a)

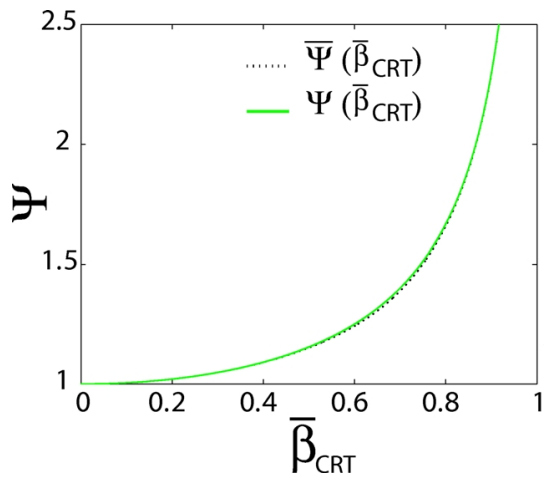

(b)

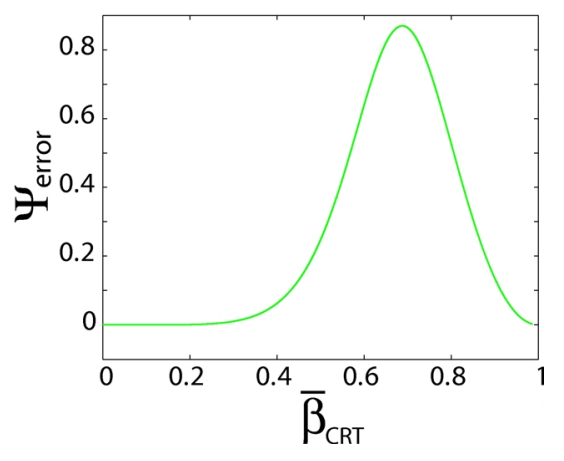

Fig. 1. Proposed model matching the apparent velocity dependence of mass in CRT experiments. (a) Comparison of apparent mass variations $\Psi\left(\bar{\beta}_{\mathrm{CRT}}\right)$ (relativistic mass model) and $\bar{\Psi}\left(\bar{\beta}_{\mathrm{CRT}}\right)$ (simplified model) as in (39), and (40) with normalized relative speed $\bar{\beta}_{\mathrm{CRT}}$ in (38). (b) The difference $\Psi_{\text {error }}$ in predicted mass variation is less than $1 \%$ with the simplified model as in (41). There would be no error with the exact relative-velocity model in (36).

Moreover, the percentage difference $\Psi_{\text {error }}$ between the two expressions (39) and (40) given by

$$
\Psi_{\text {error }}=\frac{\Psi\left(\bar{\beta}_{\mathrm{CRT}}\right)-\bar{\Psi}\left(\bar{\beta}_{\mathrm{CRT}}\right)}{\Psi\left(\bar{\beta}_{\mathrm{CRT}}\right)} \cdot 100
$$

is less than $1 \%$ as shown in Figure 1. Thus, the relativistic velocity dependency of mass in CRT experiments can be modeled using the relative-velocity approach with the perpendicular nonlinearity $\left(\mathcal{N}_{\perp}=\right.$ $\left.s_{\perp} \alpha\right)$ in the Lorentz force expression and a constant mass. The simplified expression for the perpendicular slip $\left[s_{\perp}=\bar{s}_{\perp}\right.$ with $\bar{s}_{\perp}$ defined in (37)] is used in the rest of the article.

\subsection{Kinetic Energy and Parallel Slip}

The expressions for the kinetic energy $\mathcal{E}_{\mathrm{KE}}$ and the parallel slip term $s_{\|}$are identified in this subsection by using the perpendicular slip term $s_{\perp}$.

\subsection{Relationship between Parallel Slip and Kinetic Energy}

Consider a charged particle $q$ moving along a straight line away from a stationary charged particle $Q$ at a distance $r \hat{r}$ as shown in Fig. 2 (case 1).

Taking the dot product with a small displacement $\mathrm{d} r \hat{r}$ with Newton's law on the charge $q$ yields

$$
s_{\|}(\beta) \frac{Q q}{4 \pi \varepsilon r^{2}} \mathrm{~d} r=m \frac{\mathrm{d} v}{\mathrm{~d} t} \mathrm{~d} r=\frac{m}{2} \mathrm{~d} v^{2}=\frac{m c^{2}}{2} \mathrm{~d} \beta^{2} .
$$

Dividing both sides by the parallel slip term $s_{\|}(\beta)$ and

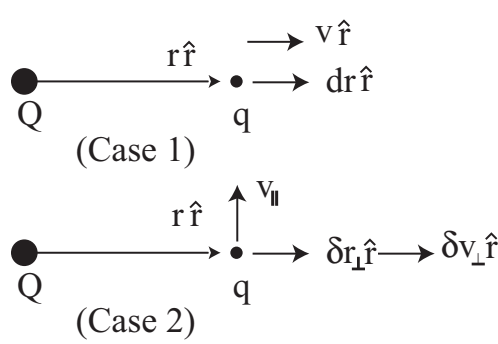

Fig. 2. Two cases: Case 1 forces parallel and case 2 forces perpendicular to the velocity.

integrating results in

$$
\int_{r_{1}}^{r_{2}} \frac{Q q}{4 \pi \varepsilon r^{2}} \mathrm{~d} r=\int_{\beta_{1}^{2}}^{\beta_{2}^{2}} \frac{m c^{2}}{2 s_{\|}(\beta)} \mathrm{d} \beta^{2}=\int_{\mathcal{E}_{\mathrm{KE}, 1}}^{\mathcal{E}_{\mathrm{KE}, 2}} \frac{\mathrm{d} \mathcal{E}_{\mathrm{KE}}}{\mathrm{d} \beta^{2}} \mathrm{~d} \beta^{2},
$$

where $\mathcal{E}_{\mathrm{KE}}$ is considered as the relative-velocitydependent kinetic energy of the system since the above expression leads to the conservation law

$$
\frac{Q q}{4 \pi \varepsilon r_{2}}+\mathcal{E}_{\mathrm{KE}, 2}=\frac{Q q}{4 \pi \varepsilon r_{1}}+\mathcal{E}_{\mathrm{KE}, 1}
$$

in which the potential energy expression $Q q /(4 \pi \varepsilon r)$ is independent of the relative velocity and the parallel slip term $s_{\|}(\beta)$. The relationship between the parallel slip term and the kinetic energy is [from (43)]

$$
\frac{\mathrm{d} \mathcal{E}_{\mathrm{KE}}}{\mathrm{d} \beta^{2}}=\frac{m c^{2}}{2 s_{\|}(\beta)} .
$$




\section{Expression for Kinetic Energy}

The perpendicular force $F_{E, \perp}$ does zero work and therefore does not lead to changes in the kinetic energy. However, an expression for the kinetic energy can be found by making the virtual work done by the perpendicular force $F_{E, \perp}$ independent of the perpendicular slip term $s_{\perp}$. Consider a charged particle $q$ moving with the velocity $v_{\|}$perpendicular to the distance vector $r \hat{r}$ from a stationary charged particle $Q$ as shown in Fig. 2 (case 2). Let $\delta r_{\perp} \hat{r}$ be a virtual displacement perpendicular to the relative velocity $v_{\|}$; then taking the dot product of the virtual displacement with both sides of Newton's law yields

$$
\begin{aligned}
s_{\perp}\left(\beta_{\|}\right) \alpha\left(\beta_{\|}\right) \frac{Q q}{4 \pi \varepsilon r^{2}} \delta r_{\perp} & =m \frac{\delta v_{\perp}}{\delta t} \delta r_{\perp}=m v_{\perp} \delta v_{\perp} \\
& =\frac{1}{2} m \delta v_{\perp}^{2}
\end{aligned}
$$

where $\beta_{\|}=\frac{\left|v_{\|}\right|}{c}$. The virtual work done can be made independent of the slip term $s_{\perp}\left(\beta_{\|}\right)$if the change in the kinetic energy $\left(\delta \mathcal{E}_{\mathrm{KE}}\right)$ has the following form [obtained by dividing both sides of the above equation by $\left.s_{\perp}\left(\beta_{\|}\right)\right]$:

$$
\begin{aligned}
\alpha\left(\beta_{\|}\right) \frac{Q q}{4 \pi \varepsilon r^{2}} \delta r_{\perp} & =\frac{1}{2} \frac{m v_{\perp}^{2}}{s_{\perp}\left(\beta_{\|}\right)}=\frac{1}{2} \frac{m c^{2} \delta \beta_{\perp}^{2}}{s_{\perp}\left(\beta_{\|}\right)} \\
& =\delta \mathcal{E}_{\mathrm{KE}}
\end{aligned}
$$

which implies that the kinetic energy $\mathcal{E}_{\mathrm{KE}}$ has the form

$$
\mathcal{E}_{\mathrm{KE}}(\beta)=\frac{1}{2} \frac{m c^{2} \beta^{2}}{s_{\perp}}=\frac{1}{2} \frac{m c^{2} \beta^{2}}{\left(1-\beta^{8}\right)^{1 / 4}} .
$$

\section{Expression for Parallel Slip Term}

Differentiating the expression (48) for the kinetic energy by $\beta^{2}$ yields

$$
\frac{\mathrm{d} \mathcal{E}_{\mathrm{KE}}}{\mathrm{d} \beta^{2}}=\frac{m c^{2}}{2}\left[\frac{1}{\left(1-\beta^{8}\right)^{5 / 4}}\right],
$$

and comparison with (45) yields the parallel slip term

$$
s_{\|}=\left(1-\beta^{8}\right)^{5 / 4} \text {. }
$$

\subsection{Summary of Relative-Velocity-Dependent Model}

The relative-velocity approach results in the following Lorentz force on an electrically charged particle [from (22) and (23)] by using the simplified slip terms [in (37) and (50)]:

$$
\begin{aligned}
F_{B}= & {\left[\left(1-\beta_{B}^{8}\right)^{1 / 4}\right] q\left(V-V_{B}\right) \times B_{\perp}, } \\
F_{E}= & {\left[\left(1-\beta_{E}^{8}\right)^{1 / 4}\right]\left[\sqrt{1-\beta_{E}^{2}}+\beta_{E}^{2}\right] q E_{\perp} } \\
& +\left[\left(1-\beta_{E}^{8}\right)^{5 / 4}\right] q E_{\|} .
\end{aligned}
$$

At low speeds, the electrical force expression (52) reduces to the expression in (20).

\section{Applications of the Lorentz Force Expression}

In this section, it is shown that the relative-velocitydependent Lorentz force expression: (a) satisfies the force between two wires (a low-velocity effect); and (b) explains the observed discrepancy in the energy of high-velocity particles.

\subsection{Force between Two Wires}

The increase in the electrical force component perpendicular to the relative velocity [in (52) which simplifies to (20) at low speeds] can be used to explain the force between two current-carrying wires, which are both stationary in a reference frame $O$. As shown in Fig. 3, let the second wire (denoted by the subscript 2) be positioned at $r \hat{r}$ from the first wire (denoted by the subscript 1). Moreover, let the currents in the two parallel wires be $I_{1}$ and $I_{2}$, and let the corresponding moving charges (per unit length) be $-\rho_{1}$ and $-\rho_{2}$ with the velocities $-v_{1} \hat{V}$ and $-v_{2} \hat{V}$, respectively, i. e.,

$$
I_{1}=\rho_{1} v_{1}, \text { and } I_{2}=\rho_{2} v_{2},
$$

where the speeds $v_{1} \geq 0$ and $v_{2} \geq 0$ of the charges are small, and $\hat{V}$ is a unit vector along the direction of the wire (in which current is flowing).

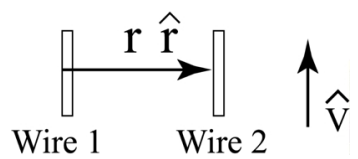

Fig. 3. Force between two currents carrying parallel wires separated by the distance $r$.

\section{Force Expression}

Consider the two (noncanceling) fields in the first wire: (a) $E_{-} \rho_{1}$ associated with the moving charge $-\rho_{1}$ with the field velocity $-v_{1} \hat{V}$ given by

$$
E_{-\rho_{1}}=\left[-\rho_{1} /(2 \pi \varepsilon r)\right] \hat{r} ;
$$


and (b) $E_{\rho_{1}}$ associated with the corresponding stationary charge $\rho_{1}$ in the wire, i. e., the stationary field given by

$$
E_{\rho_{1}}=\left[\rho_{1} /(2 \pi \varepsilon r)\right] \hat{r} .
$$

These two fields act on the moving charge $-\rho_{2}$ and a corresponding stationary charge $\rho_{2}$ on the second wire (per unit length). For example, the force per unit length $F_{-} \rho_{1},-\rho_{2}$ on the moving charge $-\rho_{2}$ due to the moving charge $-\rho_{1}$ can be obtained from (20) and (54) as

$$
\begin{aligned}
F_{-\rho_{1},-\rho_{2}} & =-\rho_{2}\left[1+\frac{\left|\left(-v_{2}\right)-\left(-v_{1}\right)\right|^{2}}{2 c^{2}}\right] E_{-\rho_{1}} \\
& =-\rho_{2}\left[1+\frac{\left|\left(-v_{2}\right)-\left(-v_{1}\right)\right|^{2}}{2 c^{2}}\right]\left[-\frac{\rho_{1}}{2 \pi \varepsilon r}\right] \hat{r} \\
& =\frac{\rho_{1} \rho_{2} \hat{r}}{2 \pi \varepsilon r}\left[1+\frac{\left|v_{1}-v_{2}\right|^{2}}{2 c^{2}}\right]
\end{aligned}
$$

Similarly, the force per unit length $F_{\rho_{1},-\rho_{2}}$ on the moving charge $-\rho_{2}$ due to the stationary charge $\rho_{1}$, as well as the the forces $F_{-\rho_{1}, \rho_{2}}, F_{\rho_{1}, \rho_{2}}$ on the stationary charge $\rho_{2}$ on the second wire due to the charges (on the first wire) $-\rho_{1}$ and $\rho_{1}$, respectively, are given by

$$
\begin{aligned}
& F_{\rho_{1},-\rho_{2}}=-\frac{\rho_{1} \rho_{2} \hat{r}}{2 \pi \varepsilon r}\left(1+\frac{\left|-v_{2}\right|^{2}}{2 c^{2}}\right), \\
& F_{-\rho_{1}, \rho_{2}}=-\frac{\rho_{1} \rho_{2} \hat{r}}{2 \pi \varepsilon r}\left(1+\frac{\left|v_{1}\right|^{2}}{2 c^{2}}\right), \\
& F_{\rho_{1}, \rho_{2}}=\frac{\rho_{1} \rho_{2} \hat{r}}{2 \pi \varepsilon r} .
\end{aligned}
$$

Thus, the total force per unit length $F_{1,2}$ on the second wire can be found using (56) and (57) as

$$
\begin{aligned}
F_{1,2}= & F_{\rho_{1}, \rho_{2}}+F_{\rho_{1},-\rho_{2}}+F_{-\rho_{1}, \rho_{2}}+F_{-\rho_{1},-\rho_{2}} \\
= & \frac{\rho_{1} \rho_{2} \hat{r}}{2 \pi \varepsilon r}\left[1-\left(1+\frac{\left|v_{2}\right|^{2}}{2 c^{2}}\right)-\left(1+\frac{\left|v_{1}\right|^{2}}{2 c^{2}}\right)\right. \\
& \left.+\left(1+\frac{\left|v_{1}-v_{2}\right|^{2}}{2 c^{2}}\right)\right] \\
= & \frac{\rho_{1} \rho_{2} \hat{r}}{2 \pi \varepsilon r}\left(\frac{-2 v_{1} v_{2}}{2 c^{2}}\right)=-\frac{\mu I_{1} I_{2}}{2 \pi r} \hat{r} .
\end{aligned}
$$

This force on the second wire is attractive (i.e., towards the first wire) if the two wires carry current in the same direction.

The Force between Wires is Incorrect with an ad-hoc Perpendicular Slip Term

Another choice of the perpendicular slip term can be found by matching the acceleration resulting from the relativistic increase in mass with speed. For example, the slip term $s_{\perp}\left(\beta_{E}\right)$ in (23) can be chosen such that the perpendicular component of the force due to an electric field becomes

$$
F_{E, \perp}=\left[s_{\perp}\left(\beta_{E}\right)\right] q \alpha E_{\perp}=q E_{\perp} \sqrt{1-\beta_{E}^{2}} .
$$

The resulting force can be approximated (at low speeds $\beta_{E}$ ) by

$$
F_{E, \perp} \cong q E_{\perp}\left(1-\frac{\beta_{E}^{2}}{2}\right) .
$$

However, this expression is not consistent with the force between two current-carrying wires. In particular, the scaling of the term $\beta_{E}^{2}$ has the opposite sign in (60) when compared to the expression in (20). The use of the expression in (60) would lead to a force

$$
F_{1,2}=\frac{\mu I_{1} I_{2}}{2 \pi r} \hat{r}
$$

similar to (58); however, the force between two wires that are carrying current in the same direction is repulsive, which is incorrect.

\subsection{High-Velocity Particles}

The energy and velocity of charged particles in magnetic fields predicted by the relative-velocity approach are compared with relativistic predictions. These are then used to explain apparent discrepancies in the measured energy of high-velocity experiments.

\section{Comparison of Energy and Velocity Expressions}

The motion of a particle with the charge $q$ and the mass $m$ moving with the speed $v$ in a magnetic field $B$ along a circle with radius $\rho$ is governed by

$$
\begin{aligned}
& \frac{m v^{2}}{\rho}=q v s_{\perp} B, \text { i. e., } \\
& \frac{\beta}{\left(1-\beta^{8}\right)^{1 / 4}}=\left(\frac{q}{m c}\right) \rho B=\kappa .
\end{aligned}
$$

The relativistic expression for the velocity $v_{\mathrm{r}}$ (and $\beta_{\mathrm{r}}=$ $\left.\left|v_{\mathrm{r}}\right| / c\right)$, for a given value of the non-dimensional parameter $\kappa$, can be found as

$$
\frac{m v_{\mathrm{r}}^{2}}{\rho \sqrt{1-\beta_{\mathrm{r}}^{2}}}=q v_{\mathrm{r}} B, \text { i. e., } \frac{\beta_{\mathrm{r}}}{\left(1-\beta_{\mathrm{r}}^{2}\right)^{1 / 2}}=\kappa,
$$


(a)

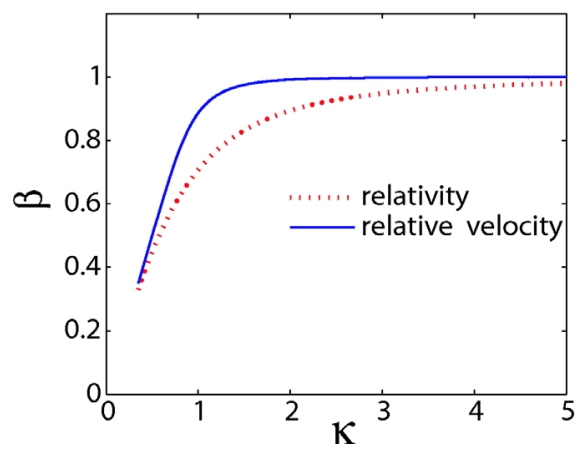

Fig. 4. Variation of (a) the normalized speed and (b) the energy.

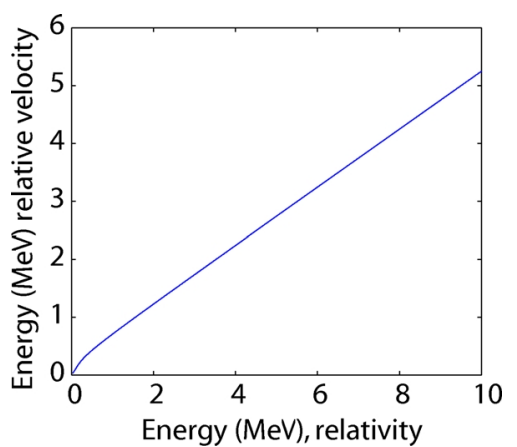

(b)

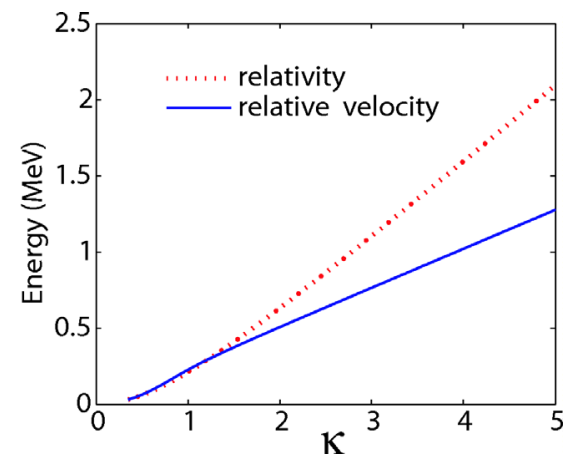

(b)

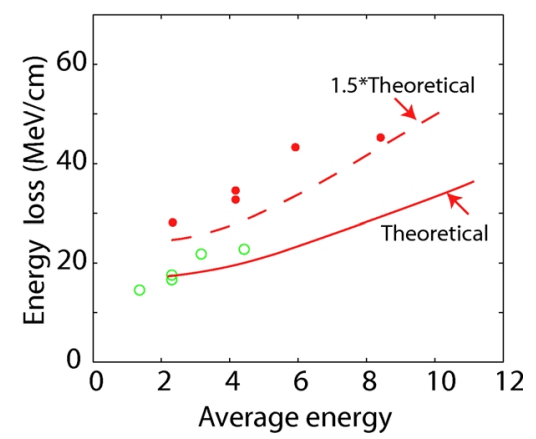

Fig. 5. (a) Relative-velocity-based energy vs. relativistic energy in magnetic fields. (b) The large deviation between the predicted and experimental energy loss $\Delta \mathcal{E}$ for different average energies $\overline{\mathcal{E}}$ is avoided with the relative-velocity-based approach. The relative-velocity-based values (circles) are from Table 1; the relativity-based values (solid dots) and the theoretical curves (solid/dashed lines) are from [3], Figure 7.

where $m$ represents the rest mass in relativity. The corresponding relativistic energy $\mathcal{E}_{\mathrm{r}}$ is given by

$$
\mathcal{E}_{\mathrm{r}}=\frac{m c^{2}}{\left(1-\beta_{\mathrm{r}}^{2}\right)^{1 / 2}}-m c^{2},
$$

while the relative-velocity-based energy $\mathcal{E}$ is given by (48). The predicted velocity and energy for the relative-velocity approach are compared with those from the relativistic expressions in Figure 4.

Note that the velocity $\beta$ predicted by the relativevelocity approach tends to be higher than the relativistic value $\beta_{\mathrm{r}}$ while the energy $\mathcal{E}$ predicted by the relative-velocity approach tends to be lower than the relativistic value $\mathcal{E}_{\mathrm{r}}$ for large values of $\kappa$. This difference is used to explain (below) the discrepancies in the observed energy in absorption experiments and in the $\beta$-ray spectrum.

\section{Absorption of High-Energy Electrons}

Crane and co-workers [3] investigated the absorption of high-energy electrons in lead by measuring the initial energy $\mathcal{E}_{\mathrm{r}, \mathrm{i}}$ and final energy $\mathcal{E}_{\mathrm{r}, \mathrm{f}}$ of electrons before and after passing through a lead absorber in a cloud chamber [3]. The subscript $r$ denotes that the relativistic expression is used to find the energy from the measured curvature in a magnetic field. The corresponding initial and final energies, from the relative-velocity approach, are found from Fig. 5a which plots the energy from the relativevelocity approach against the corresponding relativistic energy at different radii in the magnetic field (i.e., different $\kappa$ ). Data from Crane's results ([3], Fig. 7) is used to re-compute the energy loss using the relativevelocity approach. The results are shown in Table 1 and in Fig. 5b demonstrating the recomputed energy loss $\Delta \mathcal{E}=\mathcal{E}_{\mathrm{i}}-\mathcal{E}_{\mathrm{f}}$ versus the average energy $\overline{\mathcal{E}}=\left(\mathcal{E}_{\mathrm{i}}-\mathcal{E}_{\mathrm{f}}\right) / 2$.

Thus, the results show that the large deviation (more than 50\% increase) in the energy loss from the theoretical prediction ([3], Fig. 7), when energy is computed with the relativistic approach, is avoided if the data is re-interpreted with the relative-velocity-based expression for energy. 


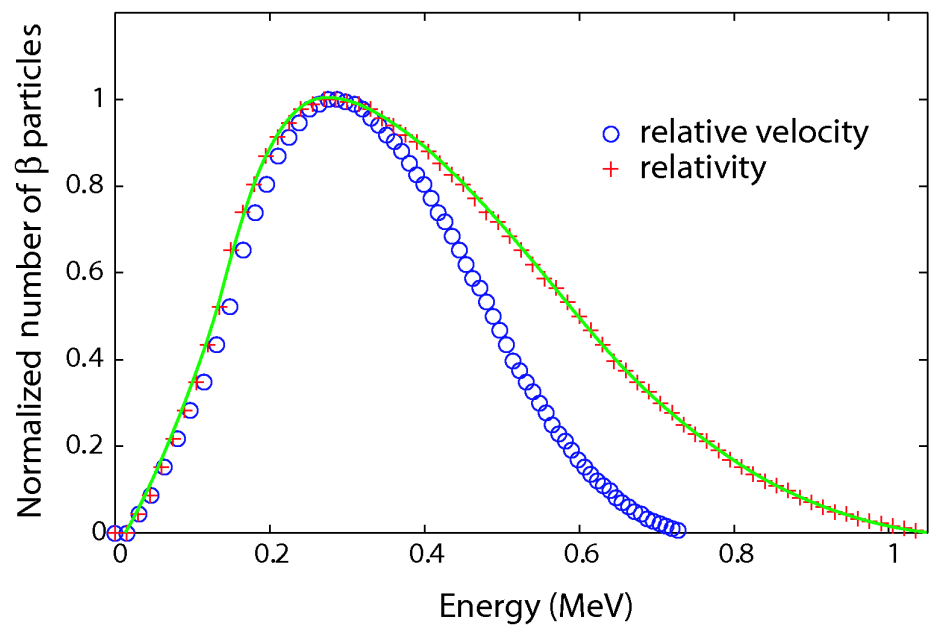

Fig. 6. $\beta$-Particle counts vs. energy. The relative-velocity approach predicts a reduction in the number of particles at the high-energy end of the spectrum; the resulting average energy is closer to the temperature-based measurement by Ellis and Wooster [4]. The solid line is Madgwick's curve (from [4], Fig. 1) with the relativistic energy expression. The crosses are data points obtained from the solid curve; these data points are used to find the corresponding relative-velocity-based energy (circles).
Table 1. Re-evaluation of energy loss in electron absorption from data by Turin and Crane [3], where the thickness of the lead absorber is $0.5 \mathrm{~mm}$.

\begin{tabular}{ccccccccc}
\hline \multicolumn{3}{c}{ Relativity } & approach & \multicolumn{4}{c}{ Relative velocity } \\
\hline Average & Loss & Initial & Final & \multicolumn{2}{c}{ Initial } & Final & Average & Loss \\
$\overline{\mathcal{E}}_{\mathrm{r}}$, & $\Delta \mathcal{E}_{\mathrm{r}}$, & $\mathcal{E}_{\mathrm{r}, \mathrm{i}}$, & $\mathcal{E}_{\mathrm{r}, \mathrm{f}}$, & \multicolumn{2}{c}{$\mathcal{E}_{\mathrm{i}}}$, & $\mathcal{E}_{\mathrm{f}}$, & $\overline{\mathcal{E}}$, & $\Delta \mathcal{E}$, \\
$\mathrm{MeV}$ & $\mathrm{MeV} / \mathrm{cm}$ & $\mathrm{MeV}$ & $\mathrm{MeV}$ & $\mathrm{MeV}$ & $\mathrm{MeV}$ & $\mathrm{MeV}$ & $\mathrm{MeV} / \mathrm{cm}$ \\
\hline 2.27 & 28.5 & 2.983 & 1.558 & 1.728 & 1.002 & 1.365 & 14.424 \\
4.12 & 33 & 4.945 & 3.295 & 2.716 & 1.886 & 2.301 & 16.606 \\
4.12 & 35 & 4.995 & 3.245 & 2.741 & 1.861 & 2.301 & 17.613 \\
5.85 & 43.5 & 6.938 & 4.763 & 3.716 & 2.624 & 3.170 & 21.823 \\
8.35 & 45.5 & 9.488 & 7.213 & 4.993 & 3.853 & 4.423 & 22.789 \\
\hline
\end{tabular}

Average Energy of the $\beta$-Ray Spectrum

Ellis and Wooster [4] found the average disintegration energy of $\mathrm{Ra} \mathrm{E}$ to be $0.344 \mathrm{MeV}$ using temperature measurements while the average energy found from the $\beta$-ray spectrum in magnetic fields ([4], Fig. 1) was $0.395 \mathrm{MeV}$. The resulting spectrum is shown in Fig. 6 (solid line); data points were measured on this curve and the corresponding relative-velocity-based energy was found using the same relationship of Figure 5a. The re-computed data points are also shown in Figure 6.

Note that the value of the energy is smaller for the relative-velocity approach when compared to the relativistic energy (towards the higher end of the spectrum in Fig. 6); therefore, the average energy is smaller with the relative-velocity approach. The average value of the spectrum with the relativistic data points is $0.39 \mathrm{MeV}$ while the average value of the spectrum with the relative-velocity-based points is $0.35 \mathrm{MeV}$. Thus, the re-computed average energy $(0.35 \mathrm{MeV})$ of Madgwick's $\beta$-ray spectrum data is close to the average $(0.344 \mathrm{MeV})$ obtained by Ellis and Wooster [4] using temperature measurements when compared to the value of $0.39 \mathrm{MeV}$ with the relativistic energy expression [4]. Therefore, the apparent discrepancy in Madgwick's data [4] can be explained by using the relative-velocity-based approach.

\section{Optics}

The field velocities $\left(V_{E}, V_{B}\right)$ introduce extra terms in Maxwell's equations that are removed to retain co-ordinate invariance. It is shown that the proposed model captures relativistic effects in: (i) the propagation speed of light; (ii) the stellar aberration; (iii) the transverse Doppler effect; and (iv) the convection of light by moving media.

\subsection{Relative-Velocity in Maxwell's Equations}

Consider an electric field $E$ and a magnetic field $B$, which are stationary with respect to an inertial frame $\mathrm{O}_{1}$ and satisfy Maxwell's equations in free space without charges:

$$
\begin{aligned}
& \nabla \times E=-\frac{\partial B}{\partial t}, \\
& \nabla \times B=\varepsilon \mu \frac{\partial E}{\partial t} .
\end{aligned}
$$

Consider the same equations in a different inertial frame $\mathrm{O}_{2}$ in which the inertial frame $\mathrm{O}_{1}$ is moving with constant velocity $V$. The Galilean transformation between the two frames

$$
X_{2}=X_{1}+V t
$$


gives the following relations at any location $\left[X_{1}\right.$ in frame $\mathrm{O}_{1}$ or $\left(X_{2}-V t\right)$ in frame $\left.\mathrm{O}_{2}\right]$ :

$$
\begin{aligned}
& \text { Frame } \mathrm{O}_{1} \text { Frame } \mathrm{O}_{2} \\
& E(a, b), \quad B(a, b), E(a, b), \quad B(a, b), \\
& a=X_{1}, \quad b=t, a=X_{2}-V t, \quad b=t, \\
& V_{E}=0, \quad V_{B}=0, V_{E}=V, \quad V_{B}=V, \\
& \frac{\partial E}{\partial t}=\frac{\partial E}{\partial b}, \frac{\partial E}{\partial t}=\frac{\partial E}{\partial a}\left(-V_{E}\right)+\frac{\partial E}{\partial b} \\
&=-\left(V_{E} \cdot \nabla\right) E+\frac{\partial E}{\partial b}, \\
& \frac{\partial E}{\partial X_{1}}=\frac{\partial E}{\partial a}, \frac{\partial E}{\partial X_{2}}=\frac{\partial E}{\partial a} .
\end{aligned}
$$

Since the spatial gradient is invariant with frame, in (73), the curl - e.g., $\nabla \times B$ on the left-hand side of (66) - is also frame-invariant. However, the partial time derivative in (72) has an extra term in frame 2 . Therefore, the partial derivative with time, such as $\frac{\partial E}{\partial t}$ on the right-hand side of (66), has an extra term $-\left(V_{E} \cdot \nabla\right) E$. Hence, adding the term $\left(V_{E} \cdot \nabla\right) E$ to Maxwell's equation (66) will make it frame-invariant under the relative-velocity-dependent approach; the modified equation becomes

$$
\nabla \times B=\varepsilon \mu\left(\frac{\partial E}{\partial t}+\left(V_{E} \cdot \nabla\right) E\right) .
$$

Noting that

$$
\frac{\mathrm{d} E}{\mathrm{~d} t}=\frac{\partial E}{\partial t}+\left(V_{E} \cdot \nabla\right) E
$$

and using a similar argument to modify (65), we obtain the following inertial-frame-invariant form of Maxwell's equations with terms that include the field velocities $V_{E}, V_{B}$ :

$$
\begin{aligned}
& \nabla \times E=-\frac{\mathrm{d} B}{\mathrm{~d} t}, \\
& \nabla \times B=\varepsilon \mu \frac{\mathrm{d} E}{\mathrm{~d} t} .
\end{aligned}
$$

\section{Invariance with Co-Ordinate Change}

Electric $E$ and magnetic fields $B$, with field velocities $V_{E}$ and $V_{B}$, respectively, that satisfy Maxwell's equations in one reference frame, also satisfy it in another inertial reference frame with a Galilean transformation of the field velocities. In this sense, the modified Maxwell's equations (76) and (77) with the total time derivatives are invariant to Galilean transformations between inertial reference frames.

\section{Addition of Current Density}

It is noted that a current density of the form

$$
\mu J=\mu \varepsilon(\nabla \cdot E) V_{E}
$$

can be added to the right-hand side of (77) but is not needed in the following discussion on optics.

\subsection{Propagation Speed of Light}

Consider the following two wave equations, which are considered as disturbances on the nominal electric field $E$ and magnetic field $B$, each of which has a field velocity

$$
V_{E}=V_{B}=V=v_{z} \hat{z}
$$

with magnitude $v_{z}$ in the $\hat{z}$-direction:

$$
\begin{aligned}
& E=e_{x} \cos (\omega t-k z) \hat{x}, \\
& B=b_{y} \cos (\omega t-k z) \hat{y} .
\end{aligned}
$$

The terms in the modified Maxwell's equations (76) and (77) for the above wave equations are computed:

$$
\begin{aligned}
& \nabla \times B=-b_{y} k \sin (\omega t-k z) \hat{x}, \\
& \nabla \times E=e_{x} k \sin (\omega t-k z) \hat{y}, \\
& \frac{\mathrm{d} B}{\mathrm{~d} t}=\left[-\omega+k v_{z}\right] b_{y} \sin (\omega t-k z) \hat{y}, \\
& \frac{\mathrm{d} E}{\mathrm{~d} t}=\left[-\omega+k v_{z}\right] e_{x} \sin (\omega t-k z) \hat{x} .
\end{aligned}
$$

Substituting (81) - (84) into the modified Maxwell's equations (76) and (77) yields

$$
\begin{aligned}
& e_{x} k=-\left[-\omega+k v_{z}\right] b_{y}, \\
& -b_{y} k=\varepsilon \mu\left[-\omega+k v_{z}\right] e_{x} .
\end{aligned}
$$

By setting $e_{x}=b_{y} c$ and $\mu \varepsilon=\frac{1}{c^{2}}$ both the equations reduce to the common expression

$$
c k=\left(\omega-k v_{z}\right) .
$$


Note that the wave propagation speed $V_{\text {light }}$ is given by $\omega / k$; therefore, the light propagation speed (in the $z$-direction) is additive, i. e.,

$$
V_{\text {light }}=\omega / k=c+v_{z}
$$

Thus, the modified Maxwell's equations allow the nominal velocity of the field $V$, in which light is generated, to be added to the standard velocity of light if the field is nonmoving - this follows directly from the invariance of the modified Maxwell's equations.

It is noted that the Michelson-Morley experiment is expected to yield the null result with the moving fields approach because the velocity of light is constant in all directions with respect to the frame of measurements (in which light was generated).

\subsection{Effect of Star's Velocity on Aberration}

In a reference frame on earth, the velocity of the earth $V_{\mathrm{e}}=v_{\mathrm{e}} \hat{V}_{\mathrm{e}}$ adds to the velocity of stellar light to generate the aberration effect, see (88), as in the original explanation by Bradley [13]. The angle of the light direction with respect to earth ( $\theta$ measured perpendicular to earth's motion as shown in Fig. 7) is maximum if the star's velocity $V_{\mathrm{s}}=v_{\mathrm{s}} \hat{V}_{\mathrm{s}}$ reduces the nominal light speed to $\left(c-v_{\mathrm{s}}\right)$ (if angle $\theta_{\mathrm{s}}=0$ ). Thus, the maximum change in the light direction with respect to earth is $2 \theta$, where

$$
\tan \theta=\frac{v_{\mathrm{e}}}{c-v_{\mathrm{s}}}
$$

For small speeds $v_{\mathrm{s}}$ and $v_{\mathrm{e}}$ the above expression is only linear in $v_{\mathrm{e}}$ (and not linear in $v_{\mathrm{s}}$ ). It can be approximated as

$$
\theta \approx \frac{v_{\mathrm{e}}}{c} .
$$

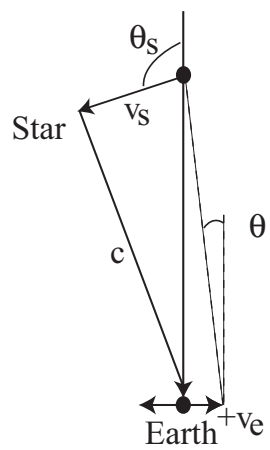

Fig. 7. Aberration formula based on relative velocity matches the classical expression [13].
The effect of the star's velocity $V_{\mathrm{s}}$ on the aberration effect (due to earth's motion) is small if the speed of the star is small, i. e., $v_{\mathrm{s}}$ is much smaller than the nominal velocity of light $c$. Therefore, stellar aberration appears to be independent of the star's velocity $V_{\mathrm{s}}[14]$ and appears to only depend on the relative change in the observer's velocity [15].

\subsection{Transverse Doppler Effect}

Consider the Doppler effect due to addition of velocities in different frames. Let light be generated in frame 1 with the velocity $C=c \hat{C}$ and angle $\theta_{1}$ (in frame 1). Moreover, in frame 2, the observed velocity of light is $C_{2}=c_{2} \hat{C}_{2}$ with angle $\theta_{2}$ as shown in Figure 8. Furthermore, let frame 1 be moving with the relative velocity $V=v \hat{V}$ relative to frame 2 .

The magnitude $c_{2}$ of the observed velocity (at an angle $\theta_{2}$ ) can be determined from the law of cosines:

$$
v^{2}+c_{2}^{2}-2 v c_{2} \cos \theta_{2}=c^{2},
$$

which implies that

$$
c_{2}=v \cos \theta_{2}+\sqrt{c^{2}-v^{2} \sin ^{2} \theta_{2}} .
$$

Hence, the frequencies $f_{1}$ and $f_{2}$ in the two frames are related by

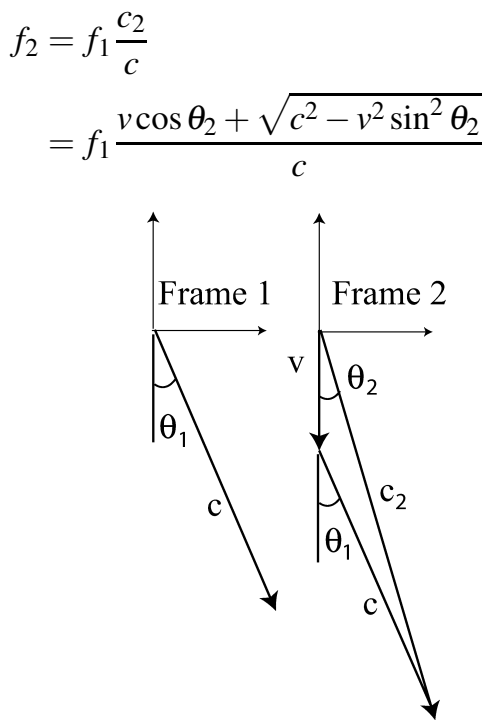

Fig. 8. Relative-velocity approach explains the transverse Doppler effect: Frame 1, which has the relative velocity $V$ with respect to frame 2 , generates light whose velocity is $c$ in frame 1 . The observed velocity of the light is at an angle $\theta_{2}$ in frame 2 . 


$$
= \begin{cases}f_{1}\left(1+\frac{v}{c}\right), & \text { if } \theta_{2}=0, \\ f_{1}\left(1-\frac{v}{c}\right), & \text { if } \theta_{2}=\pi, \\ f_{1} \sqrt{1-\left(\frac{v}{c}\right)^{2}}, & \text { if } \theta_{2}=\pi / 2 .\end{cases}
$$

The expression (92) for the transverse Doppler effect, if $\theta_{2}=\pi / 2$, matches the relativistic expression (e. g., [16], p. 301).

\subsection{Convection of Light in Moving Media}

The effect of moving media on the velocity of light through the media is shown to be similar to Fresnel's drag formula without the need for Lorentz contraction that was developed to explain this effect.

Consider a medium moving with the relative velocity $V=v \hat{V}$ in frame 1 as shown in Figure 9. For an observer $\mathrm{O}_{1}$ in frame 1 , the speed of light generated in frame 1 is $c$ (in vacuum); the goal is to estimate the effective speed of light $c_{\text {eff, }} \mathrm{O}_{1}$ through the moving media for the same observer (in frame 1). The passage of light in the moving medium can be differentiated into two types: (a) the passage of light through particles in the medium; and (b) passage through vacuum in the medium - this approach is adapted from the method by Michelson and Morley [17]. Let the mean length between particles be $L$ and the mean length of each particle be $\alpha L-$ these are measured in frame 2 that is fixed on the moving medium (as shown in Fig. 9). It is noted that the positive factor $\alpha$ tends to be small, i. e., the particle length is small when compared to the distance between particles [17].

Consider an observer $\mathrm{O}_{2}$ in frame 2 ; let the nominal speed of light through a particle in the medium be $c_{\mathrm{m}}$ if the relative velocity $V$ is zero. However, due to motion of the medium, the speed of light (generated in frame 1) through particles is $\left(c_{\mathrm{m}}-v\right)$ and through vacuum is $(c-v)$ for observer 2 . The total velocity is not a linear summation of the two velocities; the effective

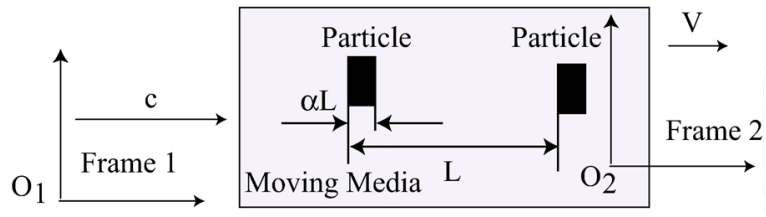

Fig. 9. Relative-velocity approach to model the convection effect (Fresnel drag) in moving media. In the moving medium (frame 2) the mean length between particles is $L$ and the average length of each particle is $\alpha L$. speed of light $c_{\text {eff, } \mathrm{O}_{2}}$ through the medium (for a fixed observer in frame $\mathrm{O}_{2}$ ) is given by

$$
\begin{aligned}
\frac{L}{c_{\text {eff }, \mathrm{O}_{2}}} & =\frac{\alpha L}{c_{\mathrm{m}}-v}+\frac{(1-\alpha) L}{c-v}, \text { i.e., } \\
c_{\mathrm{eff}, \mathrm{O}_{2}} & =\frac{1}{\frac{\alpha}{c_{\mathrm{m}}-v}+\frac{(1-\alpha)}{c-v}} .
\end{aligned}
$$

The nominal speed $c_{\text {nom }}$ of the light through the medium with zero relative velocity is obtained, by setting $v=0$ in (93), as

$$
c_{\mathrm{nom}}=\frac{1}{\frac{\alpha}{c_{\mathrm{m}}}+\frac{(1-\alpha)}{c}} .
$$

The effective velocity expression in (93) can be expanded in terms of the relative velocity $V$ as (where the higher-order terms are neglected)

$$
\begin{aligned}
c_{\mathrm{eff}, \mathrm{O}_{2}} & \approx c_{\mathrm{nom}}+\frac{-1}{\left(\frac{\alpha}{c_{\mathrm{m}}}+\frac{(1-\alpha)}{c}\right)^{2}}\left(\frac{\alpha}{c_{\mathrm{m}}^{2}}+\frac{(1-\alpha)}{c^{2}}\right) v \\
& =c_{\mathrm{nom}}-\frac{c_{\mathrm{nom}}^{2}}{c^{2}}\left(\frac{\alpha c^{2}}{c_{\mathrm{m}}^{2}}+(1-\alpha)\right) v \\
& =c_{\mathrm{nom}}-\frac{1}{\eta^{2}}\left(\frac{\alpha c^{2}}{c_{\mathrm{m}}^{2}}+(1-\alpha)\right) v,
\end{aligned}
$$

where $\eta$ is the medium's coefficient of refraction. If $\alpha$ is small, then the expression in (95) can be approximated by

$$
c_{\mathrm{eff}, \mathrm{O}_{2}} \approx c_{\mathrm{nom}}-\frac{1}{\eta^{2}} v .
$$

Rewriting in terms of observer $\mathrm{O}_{1}$ in frame 1 , by adding $v$ to the expression, leads to

$$
\begin{aligned}
c_{\mathrm{eff}, \mathrm{O}_{1}} & =c_{\mathrm{eff}, \mathrm{O}_{2}}+v=c_{\mathrm{nom}}-\frac{1}{\eta^{2}} v+v \\
& =c_{\mathrm{nom}}+\left(1-\frac{1}{\eta^{2}}\right) v
\end{aligned}
$$

which is the same as Fresnel's expression.

\section{Conclusions}

We presented a Weber-type, relative-velocity-dependent electromagnetism model. It was shown that the model: (i) captures relativistic effects in optics and high-energy particles; and (ii) explains the apparent discrepancies in experimental energy measurements. 
[1] A. K. T. Assis, Weber's Electrodynamics, Kluwer Academic Publishers, Dordrecht 1994.

[2] A. K. T. Assis and H. T. Silva, Pramana - J. Phys. 55, 393 (2000).

[3] J. J. Turin and H. R. Crane, Phys. Rev. 52, 610 (1937).

[4] C. D. Ellis and W. A. Wooster, Proc. R. Soc. London A 117, 109 (1927).

[5] E. Madgwick, Proc. Cambridge Philos. Soc. 23, 982 (1927).

[6] F. A. Scott, Phys. Rev. 48, 391 (1935).

[7] P. C. Ho and M. H. Wang, Chin. J. Phys. 2, 1 (1936).

[8] M. M. Slawsky and H.R. Crane, Phys. Rev. 59, 1203 (1939).

[9] J. R. Richardson and F. N. D. Kurie, Phys. Rev. 50, 999 (1936).
[10] L. H. Martin and A. A. Townsend, Proc. R. Soc. London A 170, 190 (1939).

[11] G. J. Neary, Proc. R. Soc. London A 175, 71 (1940).

[12] J. J. Thomson, Philos. Mag. 44, 269 (1897) (reprinted in: Classical Scientific Papers, Physics, Elsevier, New York 1964, pp. $77-100)$.

[13] J. Bradley, Philos. Trans. 35, 637 (1727).

[14] K. Brecher, Phys. Rev. Lett. 39, 1051 (1977).

[15] T. R. Phipps, Jr., Am. J. Phys. 57, 549 (1989).

[16] M. Born, Einstein's Theory of Relativity, revised edition, Dover Publication, Inc., New York 1962.

[17] A. A. Michelson and E. W. Morley, Am. J. Sci. 31, 377 (1886). 